\title{
Transition from diffuse to self-organized discharge in a high frequency dielectric barrier discharge ${ }^{\star}$
}

\author{
Antoine Belinger ${ }^{a}$, Nicolas Naudé, and Nicolas Gherardi \\ LAPLACE, Université de Toulouse, CNRS, UPS, INPT, 118 route de Narbonne, 31062 Toulouse Cedex 9, France
}

Received: 21 December 2016 / Received in final form: 2 March 2017 / Accepted: 21 April 2017

(c) EDP Sciences 2017

\begin{abstract}
Depending on the operating conditions, different regimes can be obtained in a dielectric barrier discharge (DBD): filamentary, diffuse (also called homogeneous) or self-organized. For a plane-to-plane DBD operated at high frequency $(160 \mathrm{kHz})$ and at atmospheric pressure in helium gas, we show that the addition of a small amount of nitrogen induces a transition from the diffuse regime to a self-organized regime characterized by the appearance of filaments at the exit of the discharge. In this paper, we detail mechanisms that could be responsible of the transition from diffuse mode to this self-organized mode. We point out the critical role of the power supply and the importance of the gas memory effect from one discharge to the following one on the transition to the self-organised mode. The self-organized mode is usually attributed to a surface memory effect. In this work, we show an additional involvement of the gas memory effect on the self-organized mode.
\end{abstract}

\section{Introduction}

Dielectric barrier discharge (DBD) is a discharge established in a gas gap between two electrodes whose at least one is covered by a dielectric layer [1]. Dielectric allows to limit the current of the discharge and to obtain cold plasma even at large $p d$ product ( $p$ is the pressure and $d$ the gas gap). Therefore, DBD has a lot of applications (e.g., ozone generator, excimer lamp, plasma display panel, surface treatment, flow control actuator etc.).

Usually, DBD works in the so-called filamentary mode, but depending on the gas, the electrical parameters, and the electrode configuration, a diffuse discharge can be generated. Homogeneous DBD have been obtained in helium, argon and nitrogen [2]. In nitrogen, an atmospheric pressure Townsend discharge (APTD) can be generated, while in noble gas it is an atmospheric pressure glow discharge (APGD) [3]. The nitrogen amount in a noble gas changes the regime of the discharge [4]. In any case, a Townsend breakdown generally leads to homogenous discharge [3], while a streamer breakdown leads to random filamentary discharge.

As observed in many other nonlinear systems, DBD can exhibit self-organized patterns. This phenomenon may looks like as a spatial organization of filaments in filamentary mode [5] but other patterns have been observed: strip, inversed hexagonal, concentric ring [6-8].

\footnotetext{
a e-mail: antoine.belinger@laplace.univ-tlse.fr

${ }^{\star}$ Contribution to the topical issue "The 15th International Symposium on High Pressure Low Temperature Plasma Chemistry (HAKONE XV)", edited by Nicolas Gherardi and Tomáš Hoder
}

The appearance and the dimensions of these patterns suggest that these self-organized discharges ignited with a Townsend breakdown. Recently, a hexagonal superlattice pattern has been observed, which consists on several hollow rings equally distributed on the dielectric surface [9]. Observed with short exposure time photographs, a hollow ring is the composition of periodic-driven vibrating motion of discharge filament pairs. A surface memory effect seems the cause of self-organized filaments: electric charge deposited by the previous discharge control the motions of the filaments [10]. These electric charges are deposited on the dielectrics in the same location (by the current of the discharge) or in the vicinity (by the low dark current between two filaments) of the previous discharge. It can be noted that self-organized pattern is not necessary obtain within one discharge, but successive discharges could lead to a pattern.

In any case, the self-organized denomination describes different kinds of discharges. This is certainly why it is obtained in a wide spectrum of experimental conditions: self-organized discharge pattern can thus be produced in one [11] or two dimensional discharge gap geometry [7], at low or at atmospheric pressure [7], with or without external control [12], and under a large range of temperature [13]. However, discharges leading to formation of self-organized pattern are most often produced in noble gases. As the nature of the gas changes the structure of the discharge [6], the structure of the discharge can be controlled by the composition of the gas mixture injected in the DBD [4].

In this paper, the experiments were performed in a 2D-DBD using helium and small admixture of nitrogen 
at atmospheric pressure. It is then well known that admixture of nitrogen modifies the regimes of the discharge $[4,14]$. In this work, discharge is first ignited in pure helium and remains switched on when increasing the nitrogen concentration. Thus, the regime of the discharge is controlled by the amount of nitrogen. Moreover, the atmosphere in the DBD is continually renewed by an external flow. We focus on the role of the nitrogen concentration on the self-organization and explain why the adding of nitrogen leads to a self-organized discharge. We show that both the power supply and a gas memory effect play a key role.

\section{Experimental setup}

The cell discharge is kept in a closed vessel to perform experiments in a very well controlled atmosphere. The plasma reactor is pumped down to $10^{-3}$ mbar prior to any experiment, and then it is filled up to atmospheric pressure using helium (Alphagaz 1 from Air Liquide). Then a mixture of nitrogen (Alphagaz 1 from Air Liquide) and helium is injected. Two mass flow meters allow regulating the amount of helium and nitrogen. The experimental setup is presented in Figure 1. The discharge is created between two plane electrodes, square with $3 \mathrm{~cm}$ size, each of them covered by a dielectric barrier. The high voltage (bottom) dielectric is a $600 \mu \mathrm{m}$ thick alumina plate, the grounded dielectric (top) is a $1.1 \mathrm{~mm}$ thick glass plate. So as to observe the discharge, the top electrode is transparent (thin layer of ITO).

The DBD is powered by a high frequency $(160 \mathrm{kHz})$ power supply (RFPP-LF 10) associated with a homemade matching network. The voltage is amplified using a transformer (ratio 1:20). The power supply is controlled in power and not in voltage. The electrodes are connected to the secondary of the transformer.

The high voltage applied to the DBD (V) is measured using a high voltage probe (Tektronix P6015A, pass-band $75 \mathrm{MHz}$ ), and a current coil (Pearson Electronics model 4100) measures the AC current $(I)$, with a pass-band limited to $35 \mathrm{MHz}$. The output voltage of the power supply $\left(V_{\mathrm{ps}}\right)$ is measured by a differential probe (Pintek DP 25, pass-band $25 \mathrm{MHz}$ ) and the current coming out from the power supply is measured with a second current coil (Pearson Electronics model 4100). The data are recorded on a $600 \mathrm{MHz}$ oscilloscope (Lecroy WaveSurfer 64 MXs-A).

In order to renew the atmosphere, a gas flow $(2 \mathrm{slm})$ is injected from one side of the discharge (longitudinal gas injection), keeping a constant pressure through a gentle pumping of the vessel. Two glass blocks allow to steer the flow between the electrodes and to have a $1 \mathrm{~mm}$ gas gap. The gas flow inlet consists on a slit, its size is the same that those of a transverse section of the DBD $(1 \mathrm{~mm} \times 3 \mathrm{~cm})$. The slit is located $3 \mathrm{~cm}$ before the electrode (at the entrance of the DBD cell) between the both dielectric and the glass blocks. The flow is then longitudinal to the electrode. According to the geometry of the device the mean gas velocity through the DBD is $1.1 \mathrm{~m} / \mathrm{s}$, the Reynolds number is then lower than 100. As a result, the

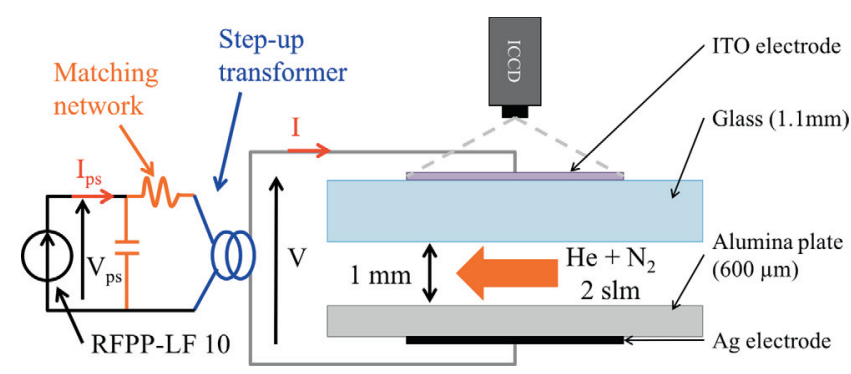

Fig. 1. Experimental setup.

flow through the DBD is laminar; there is no bulk effect on the velocity. The mean residence time of a particle in the discharge zone is $27 \mathrm{~ms}$, considering the excitation frequency, each particle see more than 8000 discharges. Even for a cross-section of $1 \mathrm{~mm}$ (typical diameter of a filament in helium), the atmosphere can be considered nearly the same between two consecutive discharges.

\subsection{Optical emission spectroscopy measurement}

Light emission of the discharge is analysed by optical emission spectroscopy (OES) using a spectrometer GETSpec 2048 TEC with a spectral range from 200 to $1100 \mathrm{~nm}$. The spectrometer is preliminary calibrated with a Deuterium Halogen light source DH-2000 from Ocean Optics. A typical spectrum is presented in Figure 2.

We focus more specifically on the emissions from $\mathrm{N}_{2}$ (2nd positive system: SPS), $\mathrm{He}$ and $\mathrm{N}_{2}^{+}$(1st negative system: FNS) shown in Figure 2.

\subsection{Discharge area measurement}

Short-exposure time photographs are taken with an intensified CCD camera (PI-MAX-3, Princeton Instruments). The ICCD camera is synchronised with the power supply. Images presented in this paper are taken principally through the top electrode.

The total area of the discharges is deduced from these pictures using an appropriate numerical filter. At first, images are smoothed with an averaging filter in order to reduce the picture noise. Afterwards, an edge filter detects the local maxima of the gradient of the image. This is followed by a broadening to close the boundaries and by erosion to detect accurately the border of the discharge. The edge filter used a canny method (edge function of the Matlab image processing toolbox with a threshold equal to 0.15). As seen in Figure 3, two kinds of discharges are observed: diffuse and filamentary. So, two different edge filters are used: a high detection threshold filter (HF) as seen in Figure 3a and a low detection threshold one (LF) as seen in Figure 3b. The difference of the two filters consists on the standard deviation of the Gaussian filter used in the canny method: 1 for the HF and 3 for the LF filter. The detected area of the discharge corresponds to the number of pixels inside the red line. As seen in Figures 3a and $3 \mathrm{~b}$, both filters do not detect the same area for the same spot. The HF filter detects only the most intensive part of the spot while the LF filter detects also the halo. 
A. Belinger et al.: Transition from diffuse to self-organized discharge in a high frequency dielectric barrier discharge

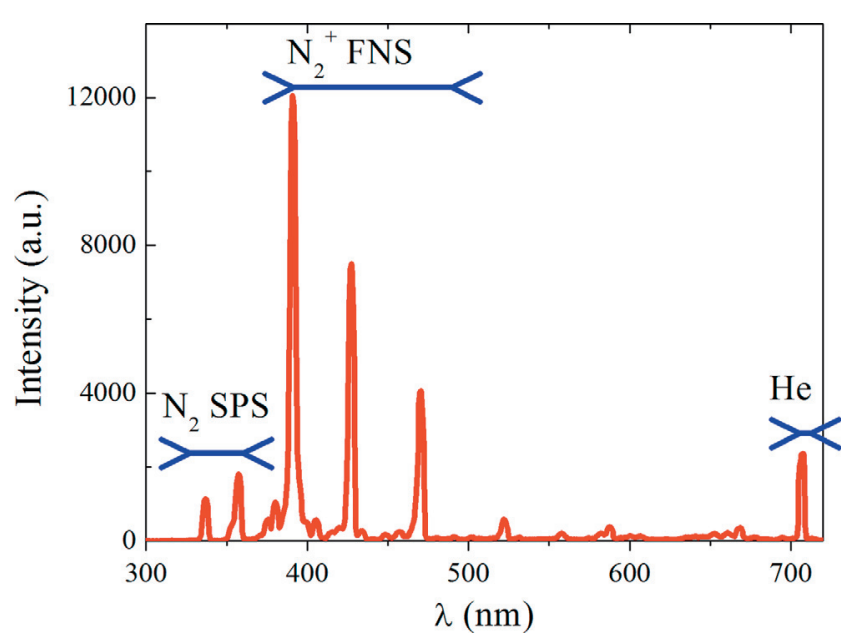

Fig. 2. Typical optical emission spectrum of the discharge. Concentration of $\mathrm{N}_{2}=0.54 \% . P=2.4 \mathrm{~W}$.

\subsection{Gas voltage calculation}

Gas current $\left(I_{\mathrm{g}}\right)$ and voltage $\left(V_{\mathrm{g}}\right)$ are calculated from the equivalent model presented on the Figure 4 and described in details in previous work [15]. $C_{\mathrm{ds}}$ and $C_{\mathrm{g}}$ are the equivalent dielectric and gas capacitance respectively. The DBD is split in two equivalent circuits: the first represents the zone without discharge (subscript 1), the second corresponds to the area of the discharge (subscript 2). So the equivalent capacitances of the DBD are split in two circuits and calculated as presented below:

$$
\begin{gathered}
C_{\mathrm{g} 1}=C_{\mathrm{g}} \times \frac{A_{\mathrm{e}}-A_{\mathrm{d}}}{A_{\mathrm{e}}}, \\
C_{\mathrm{ds} 2}=C_{\mathrm{ds}} \times \frac{A_{\mathrm{d}}}{A_{\mathrm{e}}},
\end{gathered}
$$

where $A_{\mathrm{e}}$ is the total surface of the electrode $\left(9 \mathrm{~cm}^{2}\right)$ and $A_{\mathrm{d}}$ the surface of the discharge measured from the image of the discharge. Calculation of $C_{\mathrm{g} 2}$ and $C_{\mathrm{ds} 1}$ are based on the same approach.

It is important to note that as usual with DBD, gas current and the voltage $\left(I_{\mathrm{g}}\right.$ and $\left.V_{\mathrm{g}}\right)$ depend on current and voltage applied to the $\mathrm{DBD}(V$ and $I)$ but also on the values of the capacitances $C_{\mathrm{ds} 1}, C_{\mathrm{ds} 2}, C_{\mathrm{g} 1}$ and $C_{\mathrm{g} 2}$. The area of the discharge modifies these latest capacitances. So, the precision on the discharge area measurement influences the calculation of the gas waveforms $\left(I_{\mathrm{g}}\right.$ and $\left.V_{\mathrm{g}}\right)$. For a small discharge area, the error is then more substantial than for a large area.

Figure 5 presents a result of a calculation of the current $I_{\mathrm{g}}$ and the voltage $V_{\mathrm{g}}$ for a $\mathrm{N}_{2}$ concentration of $0.5 \%$. This calculation can be used to identify the ignition voltage $V_{\text {th }}$ as shown in Figure 5 or to calculate the power or the charge transferred to the discharge. As we can see in Figure 5, during the breakdown the gas voltage $V_{\mathrm{g}}$ decreases when the current $I_{\mathrm{g}}$ increases. This is due to the formation of a cathode fall. Despite the addition of nitrogen, the discharge is diffuse and in a glow mode. It is an APGD like in pure Helium [3]. (a)



(b)

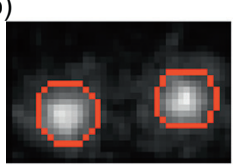

(d)



(c)

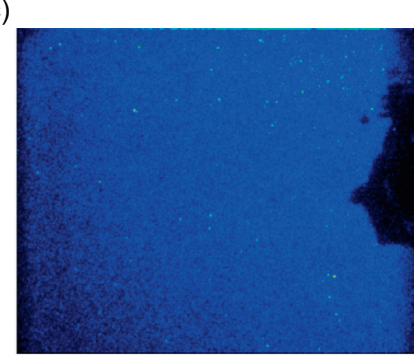

(e)

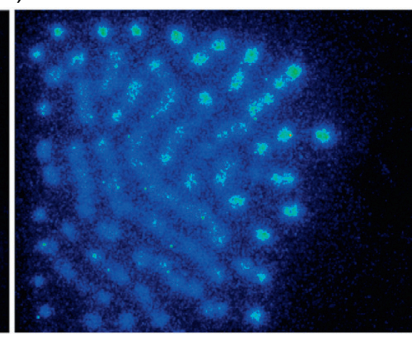

(f)
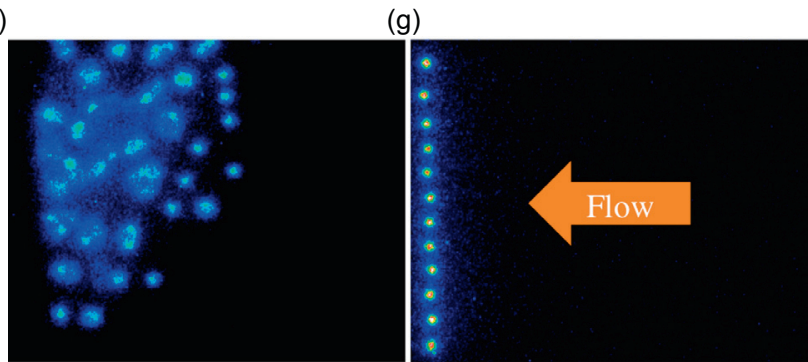

Fig. 3. Images of the discharge. (a) Spots with LF filter, (b) spots with HF filter, $(\mathrm{c}-\mathrm{g})$ increasing concentration of $\mathrm{N}_{2}$ in He, $P_{\text {start }}=2.5 \mathrm{~W}$. Exposure time is 1 period. (c) $100 \% \mathrm{He}$, (d) $1 \% \mathrm{~N}_{2}$, (e) $1.5 \% \mathrm{~N}_{2}$, (f) $1.9 \% \mathrm{~N}_{2}$, (g) $2.5 \% \mathrm{~N}_{2}$.

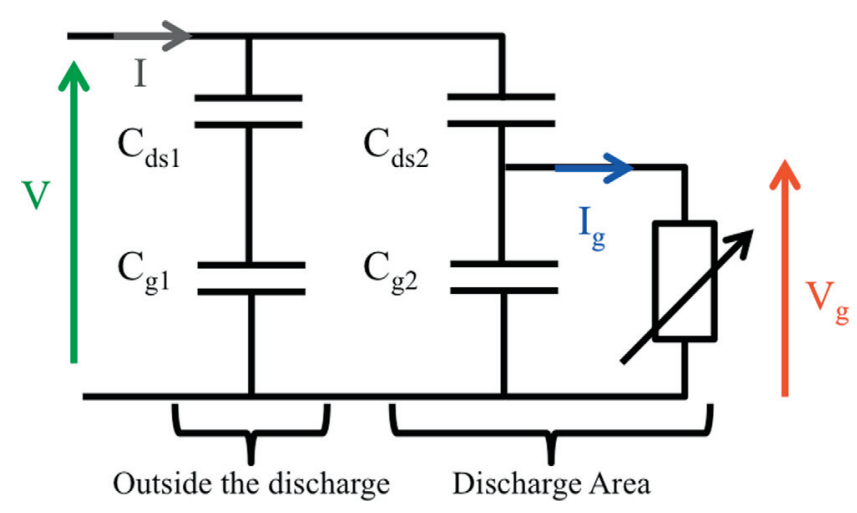

Fig. 4. Equivalent circuit of the discharge.

\section{Results and discussions}

The nitrogen proportion in the gas flow injected is increased step by step. That means that the discharge is first ignited in pure helium and remains switched on when increasing the $\mathrm{N}_{2}$. Between each concentration change, the discharge is in steady sate, and could stay in this state as long as wished. When we increase the nitrogen concentration, changes in the discharge appear very quickly 


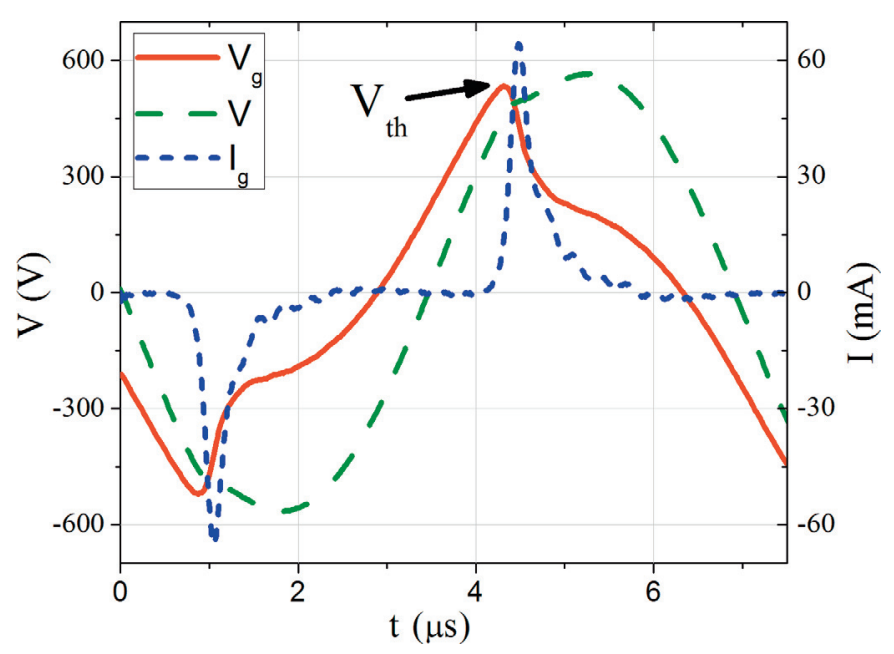

Fig. 5. Time evolution of the current $I_{\mathrm{g}}$ and the voltages $V$ and $V_{\mathrm{g}}$.

(in 1 or $2 \mathrm{~s}$ ). This is the typical time for adjustment of the gas flow from the flow controller to the discharge area. The modification of the morphologies can be hardly glimpsed by naked eye. The parameters of the power supply are unchanged during all the duration of the experiment, we noted $P_{\text {start }}$ the initial power transferred to the discharge (in pure helium). For each step, analyses are performed in steady state.

\subsection{Description of the phenomenon}

At low concentration of helium (Fig. 3c), the discharge is diffuse and spread over the whole surface of the electrodes. With the power used in Figure 3, this diffuse stage is maintained even for a concentration of $\mathrm{N}_{2}$ up to $1 \%$. The discharge area decreases but the discharge is always diffuse (Fig. 3d) and in a glow mode.

Increasing again the amount of nitrogen, some kind of microdischarges appear and coexist with a diffuse discharge, we called this mode: transition mode. As can be seen on Figures 3e and 3f, these spots appear preferentially at the exit of the DBD (along the gas flow direction). For one concentration, the area of the discharge is almost the same from one cycle to another, but the location move slowly: i.e., this displacement with no apparent rule is only observable with short exposure time picture. When $\mathrm{N}_{2}$ concentration increases again (Fig. 3g) the homogenous part of the discharge disappears suddenly, and the discharge consists on microdischarges at the exit of the DBD. These $1.2 \mathrm{~mm}^{2}$ spots are regularly spaced of $2 \mathrm{~mm}$. The microdischarges are located on a "line" at the exit of the DBD and stay fixed at the same location. We can observe them by the naked eye. For this reason, we called this mode self-organized mode (SO).

\subsection{Role of the power supply}

As observed above, the area of the discharge decreases with the nitrogen concentration. A quantification of the

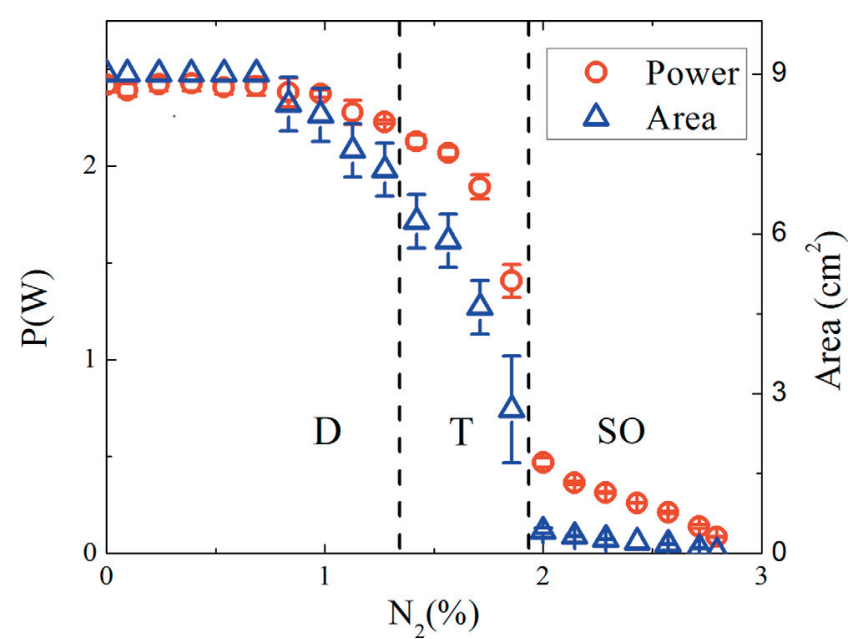

Fig. 6. Power and area of the discharge versus nitrogen concentration. D: diffuse mode, T: transition mode, SO: self-organized mode. $P_{\text {start }}=2.5 \mathrm{~W}$. In D and T mode area presented here is obtained from the LF filter, in SO area is obtained from HF filter.

area and the power transmitted to the discharge $(P)$, calculated from $I_{\mathrm{g}}$ and $V_{\mathrm{g}}$, is presented in Figure 6. The shortening of the area is related to a drop of the power transmitted to the discharge. In diffuse mode, the power decreases slowly and the power density is nearly constant $\left(0.3 \mathrm{~W} / \mathrm{cm}^{2}\right)$. In $\mathrm{SO}$ mode, the power density is also constant but higher $\left(1.2 \mathrm{~W} / \mathrm{cm}^{2}\right)$ : the reduction of the area observed in SO mode corresponds to a reduction of the number of microdischarges.

As seen in Figure 6, when the amount of $\mathrm{N}_{2}$ is higher than $0.8 \%$ the area of the discharge decreases and then the power dissipated in the discharge decreases too. It is notable that the reduction of the area affects firstly the entrance of the DBD as seen in Figure 3b. The higher the starting power is, the higher the $\mathrm{N}_{2}$ concentration is when the area of the discharge begins reducing.

Once the area of the discharge begun reducing, the equivalent capacitance of the DBD is modified. Impedance seen by the power supply is modified compared to the initial condition. Moreover, due to Paschen theory, the ignition voltage $\left(V_{\mathrm{th}}\right)$ increases with nitrogen concentration, the breakdown voltage being higher for nitrogen than for helium. As the power supply is regulated in apparent power $\left(I_{\text {rms }} \times V_{\text {rms }}\right)$ and not in voltage, the voltage of the power supply $V_{\text {ps }}$ increases. Theses both phenomena lead to a modification of the power supply operating point. The power supply mismatch can be seen in Figure 7 . At the beginning of the experiment $(100 \% \mathrm{He})$ the voltage $V_{\mathrm{ps}}$ and current $I_{\mathrm{ps}}$ of the power supply are in phase. For $1.5 \%$ of $\mathrm{N}_{2}$, the difference in phase between $V_{\mathrm{ps}}$ and $I_{\mathrm{ps}}$ moves to $52^{\circ}$, and the voltage is increased by $30 \%$. It points out the rise of the reactive power and the drop of the active power furnished by the power supply. As the power supply transmits less active power, the discharge receives less (active) power.

Before switching in SO mode, the discharge is in the glow mode, to sustain the cathodic sheath, the power 
A. Belinger et al.: Transition from diffuse to self-organized discharge in a high frequency dielectric barrier discharge

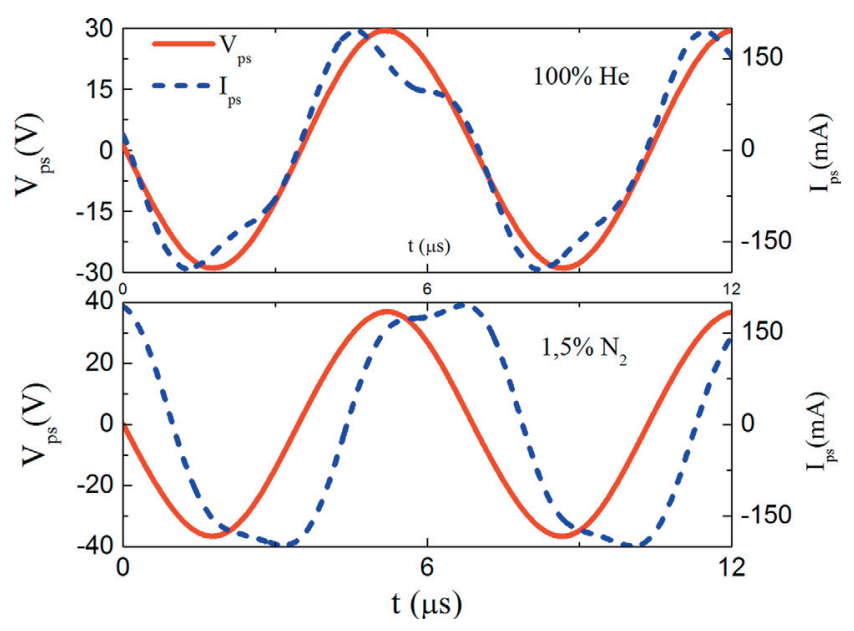

Fig. 7. Mismatch of the power supply. Voltage $V_{\mathrm{ps}}$ and current $I_{\mathrm{ps}}$ of the power supply. For $\mathrm{N}_{2}=0 \%(100 \% \mathrm{He})$ and $\mathrm{N}_{2}=1.5 \%$.

density or current density could not fall below a threshold value. In this condition, a diminution of the power implies logically a diminution of the area of the discharge. So, once the discharge area begins to reduced, due to the mismatching of the power supply the discharge area cannot raise again. The mechanisms presented above will not be observable if power supply conditions or the value of matching network component are modified during the experiment. If the power is increased after each addition of nitrogen, the discharge could stay in diffuse mode and spread on the whole surface of the DBD. Furthermore, for the initial power $P_{\text {start }}$ mentioned in Figure 6, at a concentration of nitrogen above $1 \%$, it is impossible to ignite the discharge because the voltage delivered by the power supply is too low. In order to obtain the $\mathrm{SO}$ mode with this "low power", it is mandatory to ignite the discharge at very low concentration of nitrogen and then to increase it step by step. It is also mandatory to let the power supply turned-on during the whole experiment.

The power supply operating point influences the transition to the SO mode by a mismatching that induces a drop of the transmitted power. However, the discharges observed here ensue from the previous discharges and from their "history".

\subsection{Highlighting of the memory effects}

We present in Figure 8, for a given concentration of nitrogen, the breakdown voltage $V_{\text {th }}$ measured in our conditions (when the power supply is endlessly switched-on) compared to the first breakdown voltage measured without previous discharge and therefore without memory effect. As explained in Section 2.2 the electrical parameters of the gas depend on the area of the discharge. The area is small in SO mode, then a small error in the area measurement leads to a big mistake on the $V_{\text {th }}$ estimation. As seen in Figure 3, there is a halo (detected with the LF filter) around the most intensive luminosity of the spot (detected with the HF filter). For the SO mode,

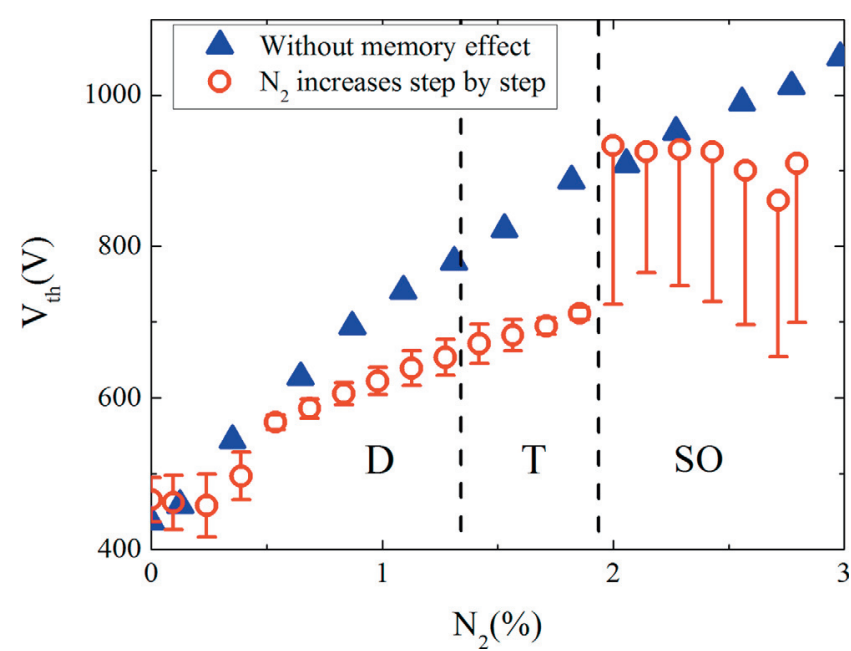

Fig. 8. Voltage ignition of the discharge $V_{\text {th }}$ with $\mathrm{N}_{2}$ increases step by step $\left(P_{\text {start }}=2.5 \mathrm{~W}\right)$ : round. Voltage ignition for the first breakdown without previous discharge (triangle).

the minimum of the error bar represents the $V_{\text {th }}$ obtained when we take into account this halo. As seen in Figure 8, the precision on the discharge area measurement influences tremendously the estimation of the breakdown voltage $V_{\text {th }}$. For the other mode, the error represents a scattering of the results.

In the experiment presented here (red circle) we can show that $V_{\mathrm{th}}$ increases with $\mathrm{N}_{2}$ amount. This rise of $V_{\mathrm{th}}$ is related to a rise of the voltage $V$ applied on the DBD. It is remarkable that it is realized without any modification on the power supply. As explained above, this autotune is allowed because the power supply is regulated in power. If the power supply was controlled by the voltage, discharge would have been switching off without a modification on the power supply.

In our experiment the power supply is never switched off, discharges occur each $3.33 \mu$ s. In Figure 8, the breakdown voltage obtained during our experiment (red circle) is lower than the one measured at the same concentration without previous discharge (blue triangle). This observation confirms that a memory effect is present and helps the ignition of the discharge.

In a helium DBD at atmospheric pressure the memory effect observed on the breakdown voltage is generally attributed to Penning ionisation of nitrogen [3]. We present here an estimation of the Penning ionisation. At atmospheric pressure, emission of the SPS of $\mathrm{N}_{2}$ (main band at $337 \mathrm{~nm}$ ) is attributed to direct electronic excitation [16]. The emission of the FNS $\mathrm{N}_{2}^{+}\left(\mathrm{B}^{2} \Sigma_{u}^{+}\right.$) (main band at $391 \mathrm{~nm}$ ) could be attributed to Penning ionisation from helium excited species $\mathrm{He}^{*}[4,16]$ but also to $\mathrm{He}_{2}^{+}$(charge transfer reaction). However, this last contribution is not important as soon as small amount of $\mathrm{N}_{2}$ is added $[17,18]$.

$$
\begin{aligned}
& \mathrm{N}_{2}+\mathrm{e}^{-} \rightarrow \mathrm{N}_{2}\left(\mathrm{C}^{3} \Pi_{u}\right)+\mathrm{e}^{-}, \\
& \mathrm{N}_{2}+\mathrm{He}^{*} \rightarrow \mathrm{N}_{2}^{+}\left(\mathrm{B}^{2} \Sigma_{u}^{+}\right)+\mathrm{e}^{-}+\mathrm{He} .
\end{aligned}
$$

Thus, the relative concentration of Helium excited species $\mathrm{He}^{*}$ can be estimated from the ratio of the emission bands, 




Fig. 9. Relative concentration of helium metastable estimated from equation (3) vs. $\mathrm{N}_{2}$ amount. $P_{\text {start }}=2.5 \mathrm{~W}$.

knowing the electron concentration. Moreover, as a crude approximation, the electron density can be estimated as proportional with the current density $J$. Hence, one can write:

$$
\left[\mathrm{He}^{*}\right]=\alpha\left[\mathrm{e}^{-}\right] \times \frac{I(391)}{I(337)} \sim J \times \frac{I(391)}{I(337)},
$$

where $J$ is calculated from the measured discharge current and from the area covered by the discharge (deduced from the pictures presented above). This method is valid under several assumptions: first of all, $\mathrm{N}_{2}^{+}$should be produced only by the helium excited-species (noted $\mathrm{He}^{*}$ ) and not by electron impact ionization (i.e., $\mathrm{N}_{2}$ concentration must be low). Secondly, the electron temperature and the electron density should be constant. This crude approximation prevents the comparison of the $\mathrm{He}^{*}$ concentration between the different regimes of the discharge.

\subsection{Importance of the helium excited species}

As seen in Figure 9, an increase of the relative nitrogen amount in the $\mathrm{He} / \mathrm{N}_{2}$ gas mixture logically results in a decrease of the $\mathrm{He}^{*}$ while the discharge stays in diffuse mode. For around $2 \%$ of nitrogen, the discharge switches in the SO mode (Fig. 3g) and it seems to be associated with a small step increase of the $\mathrm{He}^{*}$ content (the $y$-axis is logarithmic). However, one has to note here that during this transition the discharge current and power density strongly increases. It indicates a change in the discharge regime. As we explain above our approximation of $\mathrm{He}^{*}$ concentration forbids the comparison between the selforganized and the diffuse mode. This increase could be an artefact.

Experimentally in helium with admixture of nitrogen, Penning ionisation of $\mathrm{N}_{2}$ are traditionally associated to the metastable $\mathrm{He}\left(2^{3} \mathrm{~S}\right)[4,16]$ or metastable of the helium molecules as $\mathrm{He}_{2}\left(2^{3} \Sigma\right)$ [4]. By computational analysis, it is demonstrated that Penning ionisation of $\mathrm{N}_{2}$ by $\mathrm{He}_{2}$ metastable (noted $\mathrm{He}_{2}^{*}$ ) is not negligible at low concentration of $\mathrm{N}_{2}$ (few ppm) [19]. Recently, it has been experimentally shown that higher states of helium molecules (called Rydberg molecules $\mathrm{He}_{2}^{R y}$ ) are substantially present at low concentration of impurities (few ppm) [20]. Due to their high-energy level, these Rydberg molecules could also influence the penning ionisation.

For the first point of Figure 9, helium comes from the bottle and contains few impurities; we are then very close to the configuration explained in [20]. In [20], at the end of the discharge density of $\mathrm{He}_{2}^{R y}$ are higher than density of $\mathrm{He}\left(2^{3} \mathrm{~S}\right)$ and could be the main causes of Penning ionization. In the model presented in [20] $\mathrm{He}_{2}^{R y}$ and $\mathrm{He}_{2}^{*}$ have always a high density several microseconds later. Because a lake of clear coefficient rate, Penning ionization of impurities by $\mathrm{He}_{2}^{R y}$ and $\mathrm{He}_{2}^{*}$ is not taken into account, so Carbone et al. [20] does not draw hasty conclusion on the $\mathrm{He}_{2}^{R y}$ or the $\mathrm{He}_{2}^{*}$ density in the late afterglow. Lazarou et al. [19], take the same rate coefficient for the Penning ionization of $\mathrm{N}_{2}$ by $\mathrm{He}_{2}^{*}$ and by He metastable. In this condition, he shows a clear impact of the $\mathrm{He}_{2}^{*}$ between two discharges. It is then difficult to conclude which excited species is the most responsible of Penning ionization in the late afterglow. However, it is clear that most of them are not consumed after the early end of the discharge and are still present for a few microseconds. As measured in [20], $\left(\mathrm{He}\left(2^{3} \mathrm{~S}\right)\right)$ is still present few microsecond after the discharge. The lifetime of $\mathrm{He}(23 \mathrm{~S})(5.7 \mu \mathrm{s})$ in pure helium is longer than our half period $(3.3 \mu \mathrm{s})$ [21]. So, in our experiment, in pure helium $\mathrm{He}\left(2^{3} \mathrm{~S}\right)$ are probably present between two discharges.

In any case, all these He excited species (noted $\mathrm{He}^{*}$ in the rest of the paper) have long lifetimes, some of them are still present between two discharges and they can produce by Penning ionisation seed electrons before a new breakdown occurs. This allows obtaining a uniform ignition of the discharge and therefore a mostly diffuse discharge [3].

Furthermore, as seen in Figure 9, the increase of the $\mathrm{N}_{2}$ density decreases the $\mathrm{He}^{*}$ density. We can note that the nitrogen density has a clear impact on underlying mechanism of the different $\mathrm{He}^{*}$ production and destruction $[17,19] . \mathrm{N}_{2}$ consumes the $\mathrm{He}^{*}$ and reduces their lifetimes. For example, at a nitrogen concentration of $1 \%$, the lifetime of the helium metastable $\mathrm{He}\left(2^{3} \mathrm{~S}\right)$ is reduced to $50 \mathrm{~ns}$ (same calculation as [21]), which is shorter than the time between two discharges. Considering this low lifetime and the periodicity of the discharge, it could be surprising to observe a Penning effect. Nevertheless, at low frequency (some $\mathrm{kHz}$ ) in pure helium a diffuse discharge could be observed [3] and the lifetime of $\mathrm{He}\left(2^{3} \mathrm{~S}\right)$ is also much smaller than the time between two discharges. We cannot dismiss the idea that other excited species (mentioned above) could have longer lifetimes. So, in our case there is certainly enough excited species to sustain a diffuse discharge at a nitrogen concentration close to $1 \%$. In addition to metastable species, at high frequency ions trapped in the positive columns could be responsible of the memory effect [3]. Due to the ambipolar diffusion, the positive column disappearance between two discharges is 
slow and ions can still be present when the next breakdown occurs. However, on our case the gap is very small $(1 \mathrm{~mm})$ and the positive columns is not observed [22].

In the transition mode, some micro-discharges appear, according to Figure 9, $\mathrm{He}^{*}$ density is then certainly too low to sustain a diffuse discharge all over the DBD. The transition to SO mode (presented in Fig. 3g) seems to occur when the $\mathrm{He}^{*}$ becomes really too low. This is certainly why the diffuse part of the discharge seen in Figures $3 \mathrm{e}$ and $3 \mathrm{f}$ disappears and it just remains the spots located at the exit.

\subsection{Location of the discharge}

As we can see in the SO mode (Fig. 3g), spots appear preferentially at the exit of the DBD. A small increase of the nitrogen concentration is enough to eliminate the diffuse part of the discharge. This change is instantaneous and lasts less than the time for flow adjusting in steady state. In the same way, in diffuse mode the primary reduction of the discharge area occurs at the entrance. The discharge is rather located at the exit of the DBD. This phenomenon is probably due to the change of the $\mathrm{N}_{2}$ concentration inside the discharge zone related to the gas flow. Then, the $\mathrm{N}_{2}$ concentration is lower at the exit than at the entrance. The "new" gas pushed progressively the "old" gas towards the exit. The "old" gas contained some excited species $\mathrm{He}^{*}$ (responsible previously of the bulk memories effect). It is important to remind that a particle crosses the DBD in more than 8000 discharges and the lifetimes of the excited species is too low to cross the DBD (mean residence time $27 \mathrm{~ms}$ ). However, $\mathrm{He}^{*}$ are continually renewed by new discharges that are located more and more at the exit of the DBD. The entrance of the DBD is progressively depleted from excited species: the majority of the excited species are gradually located at the output of the DBD. By averaging the creating and quenching mechanisms, it is as if excited species are continually shifted towards the exit. Higher density of excited species and lower density of $\mathrm{N}_{2}$ at the output of the DBD favours the ignition at the exit.

\subsection{Memory effects in SO mode}

In Figure 10, we present the evolution of the luminosity in the vicinity of the top electrode when the discharge is in SO mode. To obtain this image, firstly we turn the camera in the flow direction (looking to the gas gap) and take short exposure time pictures of the micro-discharges (10 ns) as presented in Figure 10a. We note $Y$ the gas gap and $X$ the front side of the DBD. Secondly, for each picture we sum the intensity of three closest pixels of the bottom electrode towards the gap of the DBD: $Y$ (total gap 20 pixels). Then we plot the intensity of this sum along $X$ versus the time, we indicate the evolution of the current below. Result is presented in Figure 10b. When the current is positive the top electrode is the cathode and when the current is negative it is the anode.

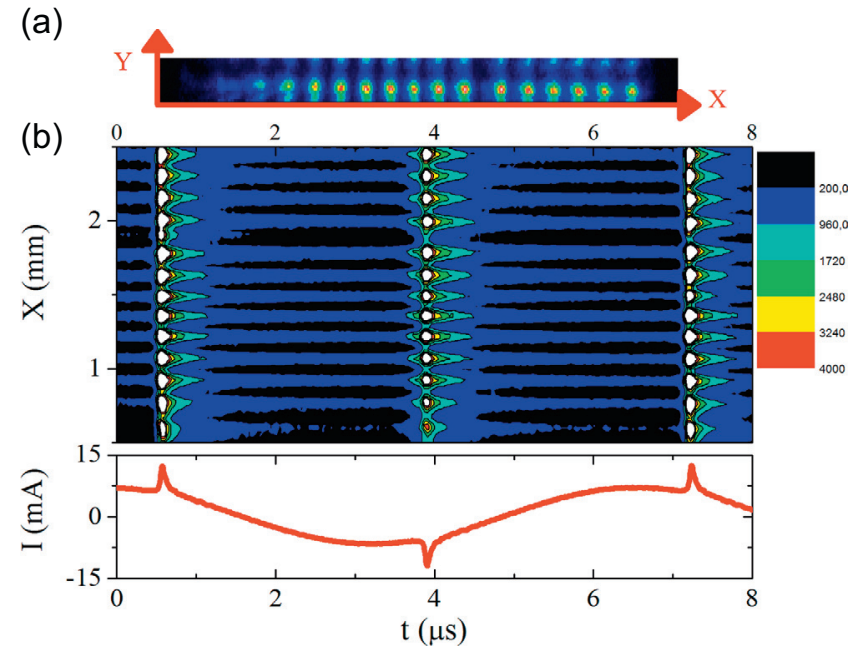

Fig. 10. (a) Image of the discharge in the flow direction. (b) Evolution of the luminosity vs. time near the top electrode with current $I$ of the DBD.

The propagation speed in the gas gap is similar for each micro discharge. It suggests that each spot represents an individual discharge event, while all events incept and develop simultaneously: each of these micro-discharges is a glow micro-discharge as presented in [22]. Furthermore, a weak luminosity stays clearly visible during the whole period where the micro discharges were ignited previously. This indicates that between two half-cycles, some excited species are still present where micro discharges occurred. This excited species could be helium atom or molecules metastables or positive ion trapped in the positive column [3] as discussed previously. In any case, the ignition of the following discharges in these areas is favored by a mechanism occurring in the gas.

According to Boeuf et al. [10] and Bogaczyk et al. [14] surface charges can also favour the ignition of the discharge. At lower frequency $(2 \mathrm{kHz})$, Bogaczyk et al. show via Pockels effect measurement that the charge is stored where the spot was located and the surface charge density stays constant until the next breakdown. The electrical model presented in Figure 4 allows calculating the charge density. In SO mode, the charge density deposited by a discharge is three times higher at position at which the discharge is located. So, in our case, there is also a surface memory effect for the micro discharge re-ignition.

With the measurements presented here, it is difficult to decide which memory effect is the strongest in SO mode. We can argue that the surface charges are fixed while the metastable move with the gas flow. Since the micro discharge is fixed in SO mode the surface memory effect is probably dominant. However, a micro-discharge has a diameter of $1 \mathrm{~mm}$. To cross it, a particle sees 270 discharges. The gas composition between two discharges is then unchanged. We can then not exclude that the bulk memories effect observed in Figure 10 also favours the fixing of the micro-discharge. We certainly observe this phenomenon because discharge occurs at high frequency compared to the renewal of the gas composition. 


\section{Conclusion}

The admixture of nitrogen in a helium APGD sustained at high frequency tends to perturb the discharge uniformity which transits to a pattern made of spots equally distributed at the exit of the DBD. Due to a high concentration of helium excited species discharge is diffuse at the lowest $\mathrm{N}_{2}$ concentrations. Adding a small amount of nitrogen, the helium metastable concentration decreases and because of the injection of external flow, is certainly lower at the entrance of the DBD. Then, it is more difficult to ignite the discharge at the entrance and the area of the discharge decreases. In the same time the ignition voltage increases. Both these phenomena tend to mismatch the power supply and then to reduce the injected power. It amplifies the reduction of the area of the discharge and some micro discharges coexist with a diffuse discharge. Contrary to many studies, here the gas or the discharge changes itself the operating point of the power supply. We observe these phenomena because the power supply is regulated in apparent power.

Afterwards, a weak but sudden increase of the nitrogen amount modifies radically the mode of the discharge. The He excited species concentration becomes too weak to sustain a diffuse discharge. The gas flow drives out to the exit the remaining metastables. So only the few micro discharges located at the exit should be maintained. These spots are visible to naked eye, there are fixed and selforganized. In this mode, the local charge deposited on the dielectric by previous discharges can sustain the discharge. However, we have shown that a gas memory effect is still present and help to fix the micro-discharges.

\section{References}

1. U. Kogelschatz, Plasma Chem. Plasma Process. 23, 1 (2003)

2. S. Okazaki, M. Kogoma, M. Uehara, Y. Kimura, J. Phys. Appl. Phys. 26, 889 (1993)
3. F. Massines, N. Gherardi, N. Naudé, P. Segur, Eur. Phys. J. Appl. Phys. 47, 22805 (2009)

4. R. Tschiersch, M. Bogaczyk, H.-E. Wagner, J. Phys. D: Appl. Phys. 47, 365204 (2014)

5. I. Brauer, M. Bode, E. Ammelt, H.-G. Purwins, Phys. Rev. Lett. 84, 4104 (2000)

6. I. Radu, R. Bartnikas, G. Czeremuszkin, M.R. Wertheimer, Plasma Sci. IEEE Trans. 31, 411 (2003)

7. W. Breazeal, K.M. Flynn, E.G. Gwinn, Phys. Rev. E 52, 1503 (1995)

8. E.L. Gurevich, A.L. Zanin, A.S. Moskalenko, H.-G. Purwins, Phys. Rev. Lett. 91, 154501 (2003)

9. B. Li, L. Dong, C. Zhang, Z. Shen, X. Zhang, J. Phys. D: Appl. Phys. 47, 055205 (2014)

10. J.P. Boeuf, B. Bernecker, T. Callegari, S. Blanco, R. Fournier, Appl. Phys. Lett. 100, 244108 (2012)

11. B. Bernecker, T. Callegari, J.P. Boeuf, J. Phys. D: Appl. Phys. 44, 262002 (2011)

12. R. Wild, T. Schumann, L. Stollenwerk, Plasma Sources Sci. Technol. 23, 054004 (2014)

13. S. Stauss, H. Muneoka, N. Ebato, F. Oshima, D.Z. Pai, K. Terashima, Plasma Sources Sci. Technol. 22, 025021 (2013)

14. M. Bogaczyk, R. Wild, L. Stollenwerk, H.-E. Wagner, J. Phys. D: Appl. Phys. 45, 465202 (2012)

15. N. Naudé, J.-P. Cambronne, N. Gherardi, F. Massines, J. Phys. D: Appl. Phys. 38, 530 (2005)

16. A. Ricard, Ph. Décomps, F. Massines, Surf. Coat. Technol. 112, 1 (1999)

17. T. Martens, A. Bogaerts, W.J.M. Brok, J. van Dijk, Appl. Phys. Lett. 92, 041504 (2008)

18. N.K. Bibinov, A.A. Fatev, K. Wiesmann, Plasma Sources Sci. Technol. 10, 579 (2001)

19. C. Lazarou, D. Koukounis, A.S. Chiper, C. Costin, I. Topala, C.E. Georghiou, Plasma Sources Sci. Technol. 24, $035012(2015)$

20. E.A.D. Carbone, C.G. Schregel, U. Czarnetzki, Plasma Sources Sci. Technol. 25, 054004 (2016)

21. B. Niermann, A. Kanitz, M. Böke, J. Winter, J. Phys. D: Appl. Phys. 44, 325201 (2011)

22. A. Belinger, N. Naudé, N. Gherardi, Plasma Sci. IEEE Trans. 42, 2816 (2014) 